abnormal in $56 \%$ cases with seizures, and $26 \%$ of those without $(\mathrm{P}<0.05)$. The group with seizures was 5 times more likely to develop new discharges at additional sites than the nonepilepsy group. In the nonepilepsy group, $46 \%$ had developmental delay, 19\% pervasive developmental disorder, and $15 \%$ had ADHD; these disorders were significantly more frequent in the nonseizure group than in those with seizures $(\mathrm{P}<0.05)$. Computerized source analysis in 12 random cases showed that isolated midline spikes were localized to the midline convexity, similar to the EEG localization by surface electrodes. (Vendrame M, Tracy M, Das R, Duffy F, Loddenkemper T, Kothare SV. Clinical correlations of midline spikes in children. Epilepsy Behav 2010;18(8):460-465). (Respond: Sanjeev V Kothare MD, Children's Hospital Boston, Fegan 9, 300 Longwood Ave, Boston, MA 02115. E-mail: Sanjeev.Kothare@childrens.harvard.edu).

COMMENT. The authors conclude that isolated midline spikes do not invariably signify an increased susceptibility to seizures, but also occur in normal children and in children with developmental and behavioral problems. A repeat EEG in a child with midline spikes and epilepsy is more likely to show new discharges in additional sites.

\title{
NEURONAL NETWORKS AND CONTINUOUS SPIKES AND WAVES DURING SLOW SLEEP
}

Haemodynamic changes associated with epileptic activity were investigated at Christian-Albrechts-University, Kiel, Germany, using simultaneous acquisitions of EEG and functional MRI in 12 children with symptomatic and cryptogenic continuous spikes and waves during slow sleep (CSWSS). MR findings were compared to electric source analysis, and all patients showed spike-related positive (activations) and negative (deactivations) blood oxygenation-level-dependent changes $(\mathrm{P}<0.05)$. The activations involved bilateral perisylvian region and cingulated gyrus in all cases, bilateral frontal cortex in 5, bilateral parietal cortex in 1, and thalamus in 5 cases. Electrical source analysis demonstrated a similar involvement of perisylvian regions, independent of the area of spike generation. Spike-related deactivations were found in precuneus, parietal cortex and medial frontal cortex in all patients and in caudate nucleus in 4 . Independent of the cause, CSWSS cases were characterized by activation of a similar neuronal network: perisylvian region, insula and cingulate gyrus. The activations correspond to both initiation and propagation pathways. (Siniatchkin M, Groening K, Moehring J, et al. Neuronal networks in children with continuous spikes and waves during slow sleep. Brain Sept 2010;133(9):2798-2813). (Respond: E-mail: m.siniatchkin@pedneuro.unikiel.de).

COMMENT. CSWSS (or electrical status epilepticus during sleep) is an age related (peak at 4-5 yrs old) epileptic encephalopathy characterized by interictal epileptic discharges during $>85 \%$ non-REM sleep, and receptive language, cognitive, and behavioral disorders. Seizures occur during sleep or while awake, partial, generalized tonic-clonic or myoclonic. CSWSS is distinguished from Landau-Kleffner syndrome by the continuous bilateral and diffuse spike-wave activity, persisting through all slow sleep

stages. (Browne, Holmes 2004) Four children with epilepsy and CSWSS, followed between 18 mos and 4 years of age, showed a pattern of behavioral and cognitive 
disturbances similar to that of autistic spectrum disorder. (Roulet Perez et al. Dev Med Child Neurol 1993;35:661-674).

\section{AUTISTIC SPECTRUM DISORDER AND CHILDHOOD ONSET EPILEPSY}

The prevalence of autistic spectrum disorder (ASD) among 519 patients with epilepsy, and the clinical characteristics of patients with both pathologies were analyzed retrospectively at Saga University, Japan. Of 79 (15.2\%) patients with epilepsy and ASD, 62 patients had idiopathic ASD and 17 had secondary ASD. Male preponderance was 47 to 15 females; median age 11 years (range 2 to 43 years). Age at onset of seizures was most frequently 4 years; $85 \%$ occurred before 10 years of age. ASD was detected after onset of epilepsy in 29 cases $(46.8 \%)$, and the diagnosis was delayed for $>5$ years in 8 , mostly high-functioning ASD cases. Complex partial seizure was the most frequent pattern, occurring in $68 \%$. Paroxysmal activities in the EEG were frontal in half the cases. Response to AEDs was complete for more than 2 years in $67.3 \%$ patients. Autistic symptoms improved after epilepsy treatment in 5 cases $(8 \%)$. (Matsuo M, Maeda T, Sasaki K, Ishii K, Hamasaki Y. Frequent association of autistic spectrum disorder in patients with childhood onset epilepsy. Brain Dev Oct 2010;32:759-763). (Respond: Muneaki Matsuo MD, Dept Pediatrics, Saga University, Japan. E-mail: matsuo@cc.sagau.ac.jp).

COMMENT. Epilepsy associated with ASD is usually characterized by complex partial seizures with frontal paroxysms with onset from 1 to 9 years of age. The prevalence of epilepsy with ASD in this study was $15 \%$, lower than some previous reports of $20 \%$ to $46 \%$ in different populations.

An International Symposium on Epilepsy in ASDs and related conditions, edited by Dr Yukio Fukuyama and colleagues, was the topic at the $12^{\text {th }}$ Annual Meeting of the Infantile Seizure Society held in Kurume, Japan, May 9-10, 2009. Several review and original articles are published in the October issue of Brain Dev 2010;32:695-780).

Dr Amy Brooks-Kayal of University of Colorado Denver School of Medicine reviews the common genetic, molecular and cellular developmental mechanisms of ASD and epilepsy. (Brain Dev Oct 2010;32:731-738). Genetic disorders sharing epilepsy and autism include tuberous sclerosis, Rett syndrome, and fragile X. Various mutations of several genes involved in neurodevelopment are found in both disorders. Early life seizures can result in cellular and molecular changes in the hippocampus that contribute to learning and behavior disorders as seen in ASD. The excitatory-inhibitory imbalance resulting from these factors may represent new therapeutic targets for treatment.

\section{NEUROPSYCHOLOGICAL SYMPTOMS AND PREDICTION OF SEIZURE RECURRENCE}

Researchers at the Departments of Medicine, Psychiatry, Neurology and Paediatrics, University of Melbourne, Australia investigated the predictive value of neuropsychiatric symptomatology in control of seizures in patients with epilepsy newly 